DOI: $10.4274 /$ tjh.galenos.2022.2021.0530

Turk J Hematol 2022;39:142-143

\title{
Mast Cell Leukemia in a Patient with Teratoma
}

\author{
Teratomlu Bir Hastada Mast Hücreli Lösemi
}

(D) Yan Shen*, (D) Zhenni Wang*, (D) Huijun Lin*, (D) Jinlin Liu

Laboratory Medicine Center, Department of Clinical Laboratory, Zhejiang Provincial People's Hospital (Affiliated People's Hospital, Hangzhou Medical College), Hangzhou, China

*These authors contributed equally to this work.
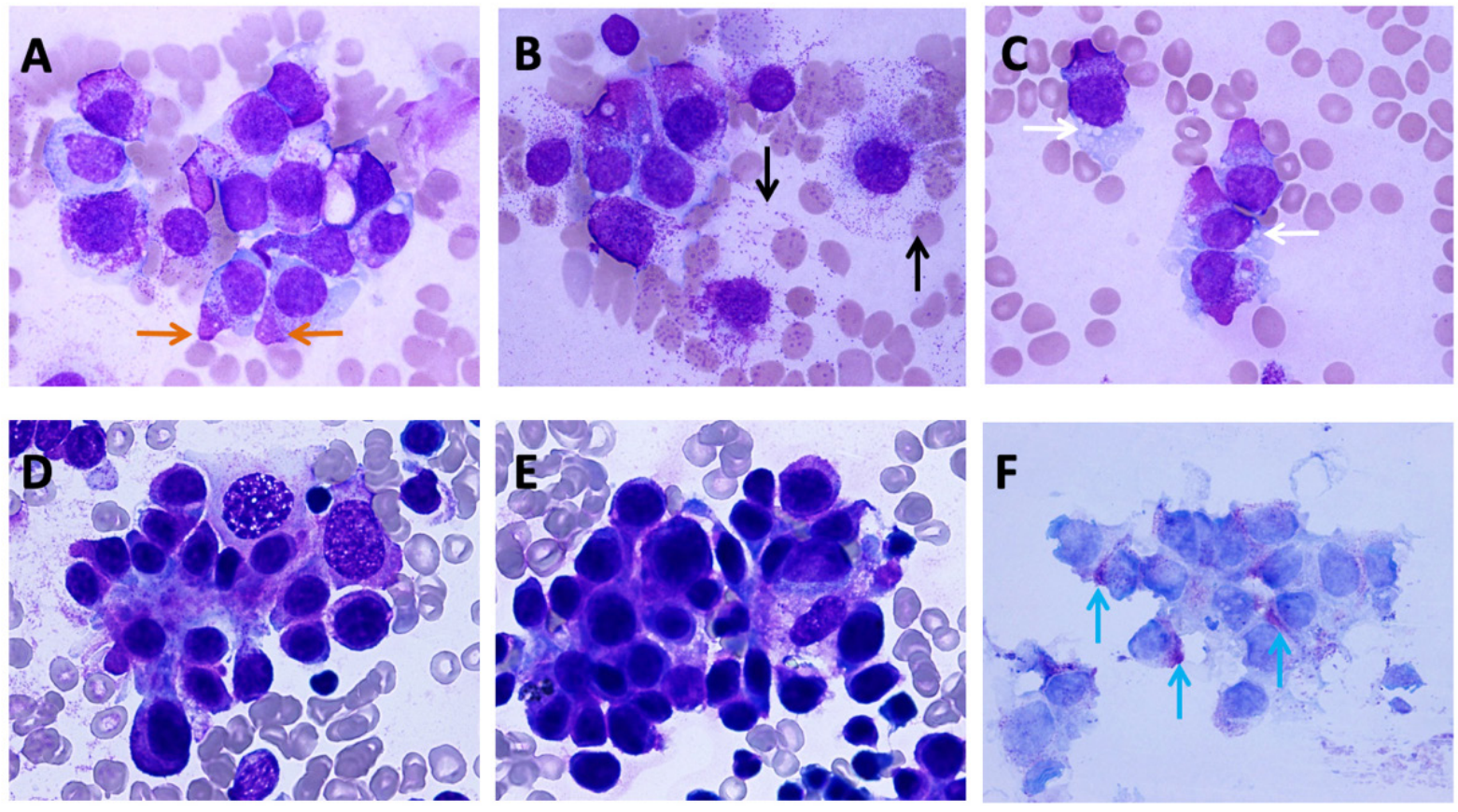

Figure 1. Blood smear showed peripheral blood involvement (40\%) by promyelocyte-like leukemia cells characterized by a spindle shape with packed and polar granule aggregates (A), dandelion-like circulating round cells (B), and prominent vacuoles in the cytoplasm (C). Bone marrow aspirate smear showed clusters of cells resembling metastatic cells $(30 \%)(D, E)$. Toluidine blue staining of the bone marrow revealed the cytoplasm to be chemoreactive for mast cells (F).

A 29-year-old man was admitted to the hospital with the complaints of left upper chest pain, asthenia, melena, and red but not itchy rash across the whole body. Initial examination for thrombocytopenia $\left(38 \times 10^{9} / \mathrm{L}\right)$, normocytic anemia $(8.2 \mathrm{~g} / \mathrm{dL})$, and white blood cell count $\left(15.6 \times 10^{9} / \mathrm{L}\right)$ yielded a diagnosis of acute promyelocyte leukemia (APL). Physical examination revealed hepatosplenomegaly.
A blood smear showed peripheral blood involvement (40\%) by promyelocyte-like leukemia cells (Figures 1A-1C). These cells were characterized by a spindle shape with packed and polar granule aggregates (Figure 1A), dandelion-like circulating round cells (Figure 1B), and prominent vacuoles in the cytoplasm (Figure 1C). A bone marrow aspirate smear showed clusters of cells resembling metastatic cells (30\%) (Figures 1D and 1E). 
Additionally, chromosome analysis revealed a normal karyotype. Considering that the patient had a history of malignant mediastinal teratoma, teratoma metastasis to the bone marrow was suspected. However, the flow cytometry for these abnormal cells was positive for CD117, CD33, CD13, CD11c, CD4, CD38, CD22, CD25, and CD2. Furthermore, toluidine blue staining of the bone marrow revealed the cytoplasm to be chemoreactive for mast cells (Figure 1F). Together with the KIT D816V mutation identified in this patient, the diagnosis of mast cell leukemia (MCL) was made according to the 2016 systemic mastocytosis criteria of the World Health Organization. Thus, this patient was treated with dasatinib, lucitanib, and dexamethasone, and later with midostaurin, but no improvement was achieved and he died two months later.

$\mathrm{MCL}$ is an aggressive form of systemic mastocytosis. Increasing promyelocyte-like blasts in the peripheral blood were initially suggestive of APL, and clusters of blast cells in the bone marrow were suggestive of the metastasis of teratoma, factors that could lead to the incorrect diagnosis of MCL. However, as atypical features were of relevance for other hematological malignancies, toluidine blue staining, immunostaining, and KIT D816V mutation were evaluated to further support the diagnosis of MCL.
Keywords: Mast cell leukemia, Teratoma

Anahtar Sözcükler: Mast hücreli lösemi, Teratom

\section{Acknowledgments}

The authors appreciate the support of the members of the Department of Clinical Laboratory, Zhejiang Provincial People's Hospital.

\section{Ethics}

Informed Consent: This study did not involve personal information; only laboratory data were reported. Patient consent was therefore waived, and this waiver was approved by the Ethics Committee of Zhejiang Provincial People's Hospital.

\section{Authorship Contributions}

Data Collection or Processing: Y.S., Z.W., H.L.; Writing: J.L.

Conflict of Interest: No conflict of interest was declared by the authors.

Financial Disclosure: This work was supported by the General Research Program of the Zhejiang Provincial Department of Health (no. 2018KY249). 